\title{
Effective Strategies for Communicating and Managing Communication in a Project Team: My Perspective
}

\author{
Brian J. Galli, Hofstra University, USA
}

iD https://orcid.org/0000-0001-9392-244X

\begin{abstract}
Projects are temporary endeavors that require a series of tasks to be completed. A project manager and their team execute these tasks. However, one of the biggest challenges that the project manager and the team will face is how to communicate effectively throughout the project. Communication is one of the most critical factors in a project's success, as without a proper communication plan and communication management strategies, a project can go downhill quickly. Also, communication is important in a business, but what makes it a crucial factor for projects is that they face deadlines and budget constraints. This paper will look at the communication process and the models that affect project environments, and it will recommend effective strategies for communicating and managing communication within project teams to aid in project success.
\end{abstract}

\section{KEYWORDS}

Communication, Project, Project Management, Team

\section{INTRODUCTION}

\section{Background}

The process of project management is to fully mobilize team members by exerting various management functions, arousing enthusiasm, and improving work efficiency to achieve project management objectives. In a sense, communication is the essence of project management, and project management is inseparable from communication (Wateridge, 1997). Moreover, communication exists in all areas and aspects of project management. In project management, communication and coordination are the bonds that carry out management in all aspects, and they are the connection among people, thoughts, information, and resources. Hence, communication is not only crucial but is also essential to the success of a project.

Project management is much different than what it used to be. Some of the impacts on this field have been beneficial, while others have hurt project management's growth. Many criteria can define the success of a project manager. Still, some that may carry the most weight include project development, cost efficiency, productivity, and projects' success rate. The project manager can tie all 
of these criteria back to the success of communication. The reasons why technology has benefited the project management industry can be seen, as online project management tools allow a team to keep its project's progress. However, the interaction between a project manager and their team is limited. Many companies and industries are heading towards the idea that working from home could be more beneficial. This is mostly due to financial health. When a team is always working separately, then the most vital piece to that team's success is communication.

Communication is how information is transmitted from the sender to the receiver, which can be among individuals or within a group, through a specific symbolic carrier that the receiver understands (Barret, 2006). Effective communication means that the information perceived by the recipient after delivery sends the same as the information by the sender, rather than the agreement between the two parties. If the recipient understands the story differently from the sender, it will lead to communication obstacles or information distortion. In project management, there are many ways to communicate, no matter how complicated. Essentially, it can always be simplified into one-to-one communication: one is the sender of information, and the other is the recipient of information. In short, communication is the process of information transfer and communication between people or organizations. Effective communication often makes the work more effective, so communication management is crucial to all stakeholders and project management success.

Communication plays an increasingly important role in projects and project success (Ksenija \& Skendrovic, 2010). Essentially, communication is exchanging anything using words and other methods to exchange thoughts, ideas, feelings, and knowledge. Furthermore, communication is a process that requires a sender and a receiver. The sender must have a message to send, and the receiver receives the message and can accept, revise, or reject the message (Rajkumar, 2010). The process also requires a medium for the message to be sent through email, face-to-face meetings, telephone, etc.

Effective communication means that the receiver understands what the sender is trying to convey. Typically, in a project environment, feedback is more complex, as it can involve more than one receiver. Thus, effective communication strategies come into play. Aside from how the communication process works, it is vital to understand the communication models when determining how to communicate and manage project teams effectively.

There are two main models used in project environments: transactional and interactive. The transactional model states that senders and receivers are both communicators, and people are connected through communication by engaging in a transaction (Schramm, 1997). It is also essential to note that this method relates to touch with social reality, cultural upbringing, and relational context (relationships). Non-verbal feedback, such as gestures and body language, is also considered feedback (Barnlund, 1970). Furthermore, the interactive communication model is based on the linear communication models, as it follows the basis of the sender channeling a message to the receiver. The receiver then becomes the sender by conducting a letter to the initial sender. The difference between this model and the transaction model is that the feedback cannot be simultaneous, and this model is best for mass communication, such as the Internet.

Communication strategies and different communication methods are the baselines of how successful a project manager will be in their career. Just as a president's legacy is defined within their first 100 days in office, a project manager's success is defined on how well they communicate to their team. What are those strategies and methods, and how can they be implemented among many different projects led by project managers?

\section{FINDINGS}

\section{The Importance of Effective Communication}

Firstly, effective communication improves team members' motivation in the project (Peterson, 2007). It enables mutual trust and support among project team members, which is also useful for 
developing relations between leadership and subordinates. Moreover, it can ease work pressure, improve interpersonal relationships, and create a harmonious working environment. In short, effective communication can eliminate obstacles, can maximize employee morale, and can make the project team more cohesive and confident.

If there is no proper communication within and outside the project, then collaborative work will not exist, and the team will never be formed. Communication not only includes the transmission of information about facts, but it also refers to everyone understanding the intent and true meaning of the information being conveyed. The project manager's responsibility is to ensure that the team is provided with the leadership, resources, tools, support, and the skills necessary to encourage innovation and commitment of teamwork. To take these responsibilities, there are many specific actions that project managers can adopt, such as creating personal images about honesty, keeping promises, and not using political skills. Managers also must encourage open and honest discussions and negotiate with team members to help form a team atmosphere.

Secondly, effective communication can improve project management efficiency (Anantatmula, 2010). The project is systematic, as it requires many people to work together, and its work efficiency is affected by each group and its team members. Effective communication helps project leaders to track the progress of the project and to adjust the work schedule. It also helps to ensure discussion, finding solutions effectively, and rapidly transferring information between project team members. Furthermore, effective communication helps develop various departments and positions within a project or organization to increase communication. To be specific, effective communication works by improving the understanding of the communication purpose to mitigate distraction from useless information, mastering the time of contact to achieve the best outcome, identifying communication project for all information to be delivered accurately, and knowing the method of communication to handle better different relations and people (Anantatmula, 2010).

Thirdly, effective communication improves the quality of project decisions (Schmidt et al., 2007). Because most projects are complex, project management would also be involved. In the process of project management, there will be many problems to solve. Hence, effective communication will enable managers to communicate with other project managers to learn advanced experience. Team members could also gain guidance from leaders, and the leaders can also attain good advice from subordinates. After the full judgment, leaders make the right decisions to solve all issues quickly and with high quality. Therefore, effective communication can help project management access more comprehensive information, which could help make the right decisions and improve the quality of decisions. Moreover, a project must first meet the customer (owner), as the project that does not meet requirements is not successful. A project should always understand the customer's needs, use the environment, and the purpose should always be communicated with the customer before the project, during the project, and after the project.

Finally, effective communication is the need for team building (Loo, 2002). A good project relies on an efficient and collaborative team. Everyone's business development and work development are inseparable from effective communication. The knowledge, experience, background, comprehension ability, and behavior of each team member would not be the same, as there should be many differences among those characters. How to eliminate these differences in the project and improve communication and collaboration between them is essential for engineering and should be considered for project managers. In life, communication is a way for managers to understand ways to enhance their emotions to improve their sense of belonging and identity, which will stabilize the team (Loo, 2002). Therefore, from the perspective of stabilizing the team and strengthening the team, regular and planned communication is an integral part of the manager's daily work.

\section{Essential Foundations of Effective Communication}

Most researchers agree that the success of a project is contingent upon the methods of communication. "The research study conducted by the Project Management Institute reveals that the use of effective 
communication methods resulted in an $80 \%$ project success rate" (Dubois, Hanlon, Koch, Nyatuga, \& Kerr, 2015). Stakeholders must be informed of all decisions to maintain successful communication. This task falls under the project manager's responsibilities, as at the beginning of a project, they should notify the team of the primary communication method. This is a significant problem in many situations due to the lack of support for a manager's strategy. If communication is not made clear from the beginning, then there will be more than one way to communicate. This will lead to significant disorganization, but as technology grows, communication methods can range from email blasts, text messages, phone calls, and face-to-face meetings. As more sophisticated project management software becomes available online, communication becomes smoother.

Another communication strategy that may persist among different teams is setting up the roles of all the stakeholders. Among the stakeholders, there will be a few key roles in which one will be the communication (Schlegel, Mcleod, \& Selfridge, 2018). The project manager's leader would be appointed and would have the main task of updating the project board and maintaining contact throughout the team, however, because there is still the possibility of a disconnect between the manager and the leader of communication. When there is miscommunication, the project manager typically has to step in and override the initial contact of the communication leader, which can confuse the team.

When most people talk about communication in project management, they tend to direct observations of previous tasks and expectations of future studies. During a project manager's meeting, the goals should also be stressed, as they usually are judges based on plans or a set of criteria (Rodriguez, 2017). Like a pyramid scheme, the goals of a project manager are reflected by the individual team members' purposes. When team members are not continuously notified of their dreams, they are less likely to complete them. Members believe that their goals have been forgotten, so reminding people of their goals and ensuring that the project management software is updated can remedy this. By communicating goals, the team members also can grasp the importance of meeting them. Many companies will award bonuses based on the number or percentage of goals a team/ project manager completes successfully.

Another strategy relates to having a concise plan. Without a system or a method of how communication will be handled, there will be chaos. Reports say that $74 \%$ of all problems in projects that go to outside sources lead to miscommunication. It is becoming more common today to have daily staff or "hub" meetings. The project manager or team leader gathers information from the stakeholders and delivers information to them.

Unfortunately, many organizations enforce better communication methods after project failure. For example, a hospital had significant communication issues between nurses and other senior staff, so people were given the wrong medicine and can cause casualties. Thus, the hospital changed its communication procedures, and every nurse got a company cell phone to maintain communication (Hansen, Lazow, \& Hagedorn, 2018). This nursing team also set up a system where one nurse would call for all of the communication to go through the one nurse. Similarly, a key stakeholder can take on being the leader of communication, but the position rotates for the nursing team. Essentially, this could be a very successful strategy, but it is contingent upon everyone participating and maintaining the will to communicate.

\section{DISCUSSION}

There are four main components to project communication management: the communication environment, the communication channel, the communication cognitive, and the communication system. Each member plays an important role when starting a project. Using the different methods and strategies from above, the four main components can be determined. 


\section{Communication Environment:}

Communication Strategy: Setting up the communication strategy is a substantial first step in becoming a successful project manager. This is a high-level overview of what needs to occur daily/weekly/ and monthly.

Organizational Structure: Determining what the proper channels are for communication. Who oversees communication? Is it the project manager, a lead communicator, or is it rotating (i.e., like the nurses in the hospital)?

\section{Communication Channel:}

Communication Methods: After determining the structure, it becomes the lead communicator's role in deciding which methods will communicate.

Communication Tools: Whatever the method may be, there will be a set of tools to go along with it. The best project managers make the best use of these communication tools.

Communication Frequency: How often will communications be distributed?

\section{Communication Cognitive:}

Communication Differences and Skills: As a project goes on, the team leader will realize that everyone has a different past when it comes to communicating with teams. Success is based on how well diversity is handled. Communication training needs may have to be established to get everyone on the same page. This can result in needing a Standard Operating Procedure of how to communicate within the team.

\section{Communication System:}

Feedback System: Most of the communication discussed stems from the top down: the project manager or communication leader is distributing all communication. A feedback system should be implemented, so that team members can communicate to upper management on the team, while still maintaining the desired organization.

Sharing and Distributing Information: This coincides with communication methods and tools but is done in greater detail. Outlining which communication methods will be used for what means is key to having concise meetings while ensuring that every team member remains in the loop.

As a successful project manager, understanding the fundamental concepts above can help set up a healthy communication plan. By understanding when and where to use each of the methods and understanding their pros and cons, a project manager can choose the best way for their team.

\section{Problems and Solutions}

All project communication stakeholders want to send and receive all information and ideas without any mistakes to achieve effective communication. However, in the actual project communication, many communication obstacles will influence project communication effectiveness, directly impact and affect the success of the project. Some problems are listed below (Stier \& Kjellin, 2010):

1. The timing of communication is improperly selected.

2. The information or ideas are incomplete.

3. Environmental impact and noise interference exist.

4. Misunderstanding or illusion exists.

5. Language and culture issues exist.

6. Non-verbal problems exist. 
Typically, it is common for ineffective communication to lead to increased costs, prevalent in many projects. For example, due to the lack of communication, two teams provide two versions of products simultaneously, but they are incompatible. Thus, the goal of the project team and the customer's needs deviate. A lot of rework and waste happens, and conflicts continue to emerge. As a result, disputes between customers and project teams develop, as the project team's internal cohesiveness declines, and even crucial team members decide to leave. Many engineers may hope to make up for their shortcomings through improving research techniques, but communication and coordination can never be replaced.

Some methods could be used to solve communication problems. Firstly, it is essential to strengthen team training and remove communication barriers (Grosse, 2002). Communication managementbased training will help with team members' communication skills, communication methods, internal communication systems, and communication responsibilities. It aims to improve communication skills and collaborative spirit. Also, it seeks to encourage each member to achieve effective communication with customers and collaborators during project implementation. In the end, everyone should continue to understand the effects and problems of communication, which will avoid the waste of time and resources. Thus, effective control of the construction period, quality, and resources can be guaranteed.

Secondly, it is essential to develop a communication plan for solving communication problems and clarify communication methods. Communication plans should be comprehensive, transparent, and streamlined. The communication plan mainly includes the following aspects (Gillard \& Johansen, 2004):

- (1) Distinguish between communication objects: Different communication objects adopt different communication methods, such as project organization structure, relevant government departments, owners, procurement suppliers, stakeholders, collaborators. The results of the analysis should be reflected in the communication plan. They should meet different people's information needs for the established communication system to be comprehensive and useful.

- (2) Differentiate communication methods: Communication methods are generally divided into written and verbal (Zulch, 2014). Oral forms, such as regular meetings, project reviews, telephone exchanges, and free discussions, are simple and effective. However, oral forms do not leave a record in black and white, such as project reports, annual reports, meeting minutes, memos, briefings, and emails. Most of the written communication is used for notifications, confirmations, and requests.

- (3) Determine the content of the communication: Communication plans should communicate the content of the information, narrow the scope of information, and reduce unnecessary information exchange (Gillard \& Johansen, 2004). To minimize differences, we should unify concepts and distinguish between different contexts and environments. At some stage of the project, team members who need assistance and support need to understand the project's progress, the quality of the project, and the operational results of the completed project.

- (4) Determine the communication time and frequency (Cleary et al., 2008): This is when it is time to communicate, how long to share, and at what stage of the project to display.

- (5) Clear communication responsibilities: This involves who is responsible for communication, what is the standard of communication, and how effective the communication is.

- (6) Establish communication feedback. Communication includes five links, such as sent, received, understood, recognized, and translated into positive behavior (Cleary et al., 2008). To achieve communication, communication management should pay attention to communication feedback and establish a communication-feedback model.

- (7) Revise the communication plan: During the project, the communication plan should be continuously updated and refined based on the information returned from the communication. It is essential to conduct a performance evaluation of communication work, encourage and supervise adjustment work, and improve communication. 
- (8) Summary of experience and lessons: For the completion of a project or the completion of a phased project, it is necessary to summarize the project work on time, exchange experiences, find deficiencies, analyze the lessons, and provide essential information and suggestions for the future performance of the project. The lessons learned need to be edited, formatted, and officially archived throughout the project's life.

Finally, it is crucial to establish a communication system and create a communication atmosphere (Gillard \& Johansen, 2004). To ensure the effective implementation of the communication plan, we must have institutional guarantees, use the system to constrain the team members, and use the system to supervise the communication. For example, the method and principle of project communication are clearly defined in the project charter. The principles that should be mastered in touch internalize the principles of communication into the organizational culture and implement them into members' actions (Gillard \& Johansen, 2004). This will create a good communication atmosphere, starting from the challenging environment and improving members' skills. Also, it will enhance the form and means of communication. Let the organization members grasp the first-hand information of the project as much as possible, such as preparing the project scope statement and continuously enriching the internal information network.

\section{EFFECTIVE STRATEGIES}

Ineffective communication contributed to $30 \%$ of project failures and was found by the Management Institute to negatively influence project success by $50 \%$ (PMI, 2017). Thus, project managers must ensure that their strategies are effective. Five main strategies aid ineffective communication for communicating and managing project teams.

\section{Strategy One: Create a Communication Plan}

All project managers have a basic communication plan for their projects, but no project or project team is the same. According to PMBOK, a communication plan is a component of the project management plan that describes how project communication will be planned, structured, implemented, and monitored for effectiveness (PMI, 2017). The plan should include multiple ideas, such as managing and communicating with stakeholders and team members, channels of communications, escalation processes, and the frequency of meetings and project updates. The plan should be so detailed that everyone who receives it knows about objectives, those involved in the project, and what they need to be doing to achieve project success.

\section{Strategy Two: Meet Regularly}

As mentioned in strategy one, the communication plan should convey how often the project team should meet and how they will meet (i.e., face-to-face meetings, teleconferences, or video conferencing). These meetings should be held frequently to keep everyone aware of project status, changes, and activity updates. Also, sessions should be between 30-45 minutes long, and each meeting should have minutes recorded.

\section{Strategy Three: Active Listening}

Although it may seem minor in the grand scheme, effective communication depends on project managers, team members, and stakeholders to actively listen to the project information. In the business world, successful project managers apply active listening to communication strategies and encourage their staff to introduce new concepts and the flow of new ideas (Chad, 2012). Furthermore, project 
managers and team members should ensure that the information is repeated to them accurately. Also, a response should be requested as a second measure of assurance that the receiver was actively listening.

\section{Strategy Four: Team Building}

Project teams can be temporary, but there are times when projects go on for years. Thus, the team will have to communicate effectively for a while if they wish to unison. Team building by project managers requires asking team members for their participation (Mir, 2013). Additionally, project success hinges on a team's ability to work and communicate effectively (Mir, 2013). In the fast-paced business field, leaders ask team members at all levels to do more with less to accomplish effective communication (Mignerat, 2012).

\section{Strategy Five: Implementation of Communication Plan \& Strategies}

It is common for project teams to create communication plans and not to implement them. However, the project manager's responsibility is to oversee that his/her team is following the communication plan and actively participating in the strategies to ensure effective communication. This might be the most challenging strategy, especially for a large group. Ensuring that the communication plan is implemented is possible by verifying that all-important communication activities are included in the project schedule. This can also consist of the resources that have been identified as necessary to carry out the communication activity, and they must be reported against at regular intervals, perhaps at project meetings (Bourne, 2010).

\section{CONCLUSION}

\section{Recommendations}

From the research conducted, it is recommended that project teams employ the transactional model for communicating. It is unrealistic to say that one strategy would work better than the next when deciding which to use. However, operating a mixture of all methods mentioned in this paper will help develop successful communication and manage communication within project environments. Furthermore, there should be careful consideration with multiple revisions to ensure that all essential personnel and stakeholders will be in the loop. Implementation is crucial because, without it, the project can run into communication issues and result in project failure. For the modes of communication within projects, respondents indicated that face-to-face communication is their preferred communication medium for initial communication regarding project issues. This was followed by telephone calls with messages in the third place, but respondents preferred that further follow-ups be done either through a face-to-face communication or by email (Smit et al., 2017).

\section{CONCLUSION}

As a project manager, it is necessary to undertake the communication link of project management. Project manager communication skills are the main factors affecting the management and leadership of the core project team. Hence, the project manager should not be buried in technology and management work. They need to spend more time communicating with project stakeholders to accurately look at the entire project from a higher level and pass the customer's intentions to the project stakeholders. At the same time, they can promptly understand the project-related personnel's needs to reach a consensus. Effective communication between the supervisors, subordinates, and project management team personnel can significantly stimulate the project. It can also improve employee enthusiasm, the entire project's efficiency, and the quality of project decisions. At the same time, it makes managing the task easier for the project manager. 
Overall, planning communication from the start will help all team members and stakeholders become more engaged. Project teams who actively listen, meet regularly, and engage in team-building exercises tend to work better together and communicate efficiently. Project managers can track the team to see if they follow the communication plan, but the process does not end at implementation. Project managers must monitor the communication plan through each stage of the project lifecycle to see if it suits the team and if it needs to be revised. Communication is about eighty to ninety percent of a project manager's job, and consideration of effective communication strategies should always be a top priority.

There are multiple aspects to constructing a successful end product. For example, team communication and the proper management of the team lead to a successful outcome. A project is likely to fail without communication strategies. According to Conant, within the industry, the "bottom-line" changes, but success in the workplace is dependent on success in the marketplace (2015). This is a shared trait amongst all companies because success in the market results from an excellent performance. Essentially, success is dependent on concise communication and management during a project life cycle. 


\section{REFERENCES}

Anantatmula, V. S. (2010). Project manager leadership role in improving project performance. Engineering Management Journal, 22(1), 13-22. doi:10.1080/10429247.2010.11431849

Barnlund, D. C. (1970). A transactional model of communication. In Foundations of Communication Theory. Harper and Row.

Bourne, L. (2010). Beyond reporting: The communication strategy. Paper presented at PMI® Global Congress. Asia Pacific, Melbourne, Victoria, Australia.

Chad, P. (2012). The use of team-based learning as an approach to increased engagement and learning for marketing students: A case study. Journal of Marketing Education, 34(2), 128-139. doi:10.1177/0273475312450388

Cleary, S., Harran, M., Luck, J., Potgieter, S., Scheckle, E., Merwe, R. D., \& Heerden, D. V. (2008). Communication: A hands-on approach. Juta \& Co. Ltd.

Dubois, M., Hanlon, J., Koch, J., Nyatuga, B., \& Kerr, N. (2015). Leadership styles of effective project managers: Techniques and traits to lead high-performance teams. Journal of Economic Development, Management, I.T. Finance and Marketing, 7(1), 30-46.

Gillard, S., \& Johansen, J. (2004). Project management communication: A systems approach. Journal of Information Science, 30(1), 23-29. doi:10.1177/0165551504041675

Grosse, C. U. (2002). Managing communication within virtual intercultural teams. Business and Professional Communication Quarterly, 65(4), 22-38. doi:10.1177/108056990206500404

Hansen, J. E., Lazow, M., \& Hagedorn, P. A. (2018). Reducing interdisciplinary communication failures through secure text messaging: A quality improvement project. Pediatric Quality \& Safety, 3(1), e053. doi:10.1097/ pq9.0000000000000053 PMID:30229189

Ksenija, C., \& Skendrovic, V. (2010). Communication management is critical for project success. Information (Basel), 43(3), 228-235.

Loo, R. (2002). Journaling: A learning tool for project management training and team-building. Project Management Journal, 33(4), 61-66. doi:10.1177/875697280203300407

Mignerat, M., \& Rivard, S. (2012). The institutionalization of information system project management practices. Information and Organization, 22(2), 125-153. doi:10.1016/j.infoandorg.2012.01.003

Mir, F. A., \& Pinnington, A. H. (2013). Exploring the value of project management: Linking project management performance and project success. International Journal of Project Management, 32(2), 202-217. doi:10.1016/j. ijproman.2013.05.012

Peterson, T. M. (2007). Motivation: How to increase project team performance. Project Management Journal, 38(4), 60-69. doi:10.1002/pmj.20019

Project Management Institute. (2017). Project Management Body of Knowledge (PMBOK®). Project Management Institute.

Rajkumar, S. (2010). Art of communication in project management. Paper presented at PMI, Research Conference: Defining the Future of Project Management, Washington, DC.

Rodriguez, P. A. (2017). Conceptual model of communication theories within the project process. INNOVA Research Journal, 2(3), 42-51. doi:10.33890/innova.v2.n3.2017.131

Schlegel, E. F., Mcleod, K. B., \& Selfridge, N. J. (2018). Practical tips for successful implementation of educational innovations: Project Management Tools for Health Professional Educators. MedEdPublish, 7(2). Advance online publication. doi:10.15694/mep.2018.0000105.1

Schmidt, J. B., Weiss, M. M., \& Massey, A. P. (2007). New product development decision-making effectiveness: Comparing individuals, face-to-face teams, and virtual teams. Decision Sciences, 32(4), 575-600. doi:10.1111/j.1540-5915.2001.tb00973.x

Schramm, W. L., Chaffee, S. H., \& Rogers, E. M. (1997). The beginnings of communication study in America. Sage. 
Smit, M. C., Bond-Barnard, T., Steyn, H., \& Fabris-Rotelli, I. (2017). Email communication in project management: A bane or a blessing? South African Journal of Information Management, 19(1). Advance online publication. doi:10.4102/sajim.v19i1.826

Stier, J., \& Kjellin, M. S. (2009). Communicative challenges in multinational project work: Obstacles and tools for reaching common understandings. Journal of Intercultural Communication, 24, 1-12.

Wateridge, J. (1997). Training for IS/IT project managers: A way forward. International Journal of Project Management, 15(5), 283-288. doi:10.1016/S0263-7863(96)00085-3

Zulch, B. G. (2014). Communication: The foundation of project management. Bloemfontein. Procedia Technology, 16, 1000-1004. doi:10.1016/j.protcy.2014.10.054

Brian J. Galli works as an Assistant Professor of Industrial Engineering \& Engineering Management in the Fred DeMatteis School of Engineering \& Applied Science at Hofstra University. He also currently serves as the Graduate Director for the Engineering Management Master degree program in the school. He has previously served as faculty at other academic institutions, including: Long Island University, New York Institute of Technology (NYIT), American Public University (APUS), SUNY Binghamton, and SUNY Stony Brook. Prior to joining academia, he worked in industry for over 12 years in which he applied industrial engineering, project management and continuous improvement in a wide variety of arenas, including healthcare, manufacturing, transactional, and service environments. He also provides project management support and training in the areas of process improvement, project management, and analytics. 